\title{
Architecture Design of an Integrated Communication and Broadcasting Network
}

\author{
Weidong Liu, Jiang Wu, Hong Shen \\ Tsinghua National Laboratory for Information Science and Technology, \\ Department of Computer Science and Technology, Tsinghua University, Beijing, China \\ E-mail: wujiangthu@gmail.com \\ Received September 17, 2010; revised September 25, 2010; accepted September 26, 2010
}

\begin{abstract}
Due to the power limitation of nodes in wire-less sensor networks (WSNs), how to maximize network lifetime has become a critical issue for deployment of WSNs. Although several schemes have been proposed for 2D WSNs, few for 3D WSNs are known. In this paper, we present a scheme to maximize network lifetime for 3D WSNs through balancing energy consumption, as an extension of the existing scheme for 2D WSNs proposed recently [1]. Same as [1], we formulate the energy consumption balancing problem as an problem of optimal distribution of transmitting data by combining the techniques of sphere-corona based network division, mixed-routing and data aggregation. We first present a Tiled-block based routing scheme in order to balance energy consumption among nodes in each sphere-corona. Then we design an algorithm to compute the optimal distribution ratio of transmitting data between direct and hop-by-hop transmission, with the purpose of balancing energy consumption among nodes across different sphere-coronas. We show maximizing network lifetime through computing the optimal number of sphere-coronas. Afterwards a energy consumption balanced data collecting protocol (ECBDC) is designed and a solution to extend ECBDC to largescale WSNs is also presented. Simulaiton results show that ECBDC is superior to conventional direct and multihop transmission schemes in network lifetime.
\end{abstract}

Keywords: Wireless Sensor Networks, 3D, Network Lifetime, Energy Balancing

\section{Introduction}

Wireless sensor networks (WSNs) have been widely used in various applications, such as environmental and industrial monitoring [2]. The ability to withstand harsh environmental conditions and to run without manual intervention is the most attractive feature for users.

The greatest difficulty for WSNs applications probably is hardware constraints: limitation in power, insufficient in computational capacity, and shortage in memory [3]. The limitation of energy supply directly influences thelifetime of network which is the critical problem for WSNs. How to reduce energy consumption to prolong network lifetime remains a key issue for WNSs. There have been rich literatures discussing this issue and numerous schemes have been proposed. Geographical adaptive fidelity (GAF) algorithm [4] conserves energy by identifying nodes that are equivalent from a routing perspective and then turning off unnecessary nodes, keeping a constant level of routing fidelity. In [5-12], minimum energy routing problems have been addressed, in order to prolong network lifetime. The approach in those works is to minimize the total energy consumed to reach the sink node, which minimizes the energy consumed by each packet or data flow.

Basically, energy consumption in sensor nodes is divided in three fields: sensing, communicating (transmitting data and receiving data) and data processing [13]. But it is necessary to notice that, in WSNs, to transmit one bit data at short range consumes much more energy than to process it [14]. Thus, most algorithms designed for WSNs aims to reduce communication cost of whole network, which can prolong network lifetime. Many other ideas are also attractive to maximize network lifetime. Three common approaches are effective to achieve maximization of system lifetime: mix-routing [15-18], network partition [1] and data aggregation [19-24].

Mix-routing scheme allows node to transmit data between direct transmission and hop-by-hop transmission. In direct mode node transmits data directly to sink node 
while transmitting data to next hop node in hop-by-hop mode. Energy consumption balance may be achieved if node chooses an appropriate data allocation in these two transmission modes, and then maximization of network lifetime could be achieved accordingly.

Network partition has great influence on network lifetime. A good dividing method may reduce the amount of data transmitted, save energy of sensor nodes, and then prolong the system lifetime. Slice-based partition and corona-based partition are two common methods for network partition. Slice-based partition divide circular network into slices. And Corona-based partition method divides circular network area into coronas centred with sink node with different radius.

Data aggregation is a useful technique to eliminate redundant data of network. Nodes in small area are close to each other and may generate redundant sensing data in WSNs like temperature monitoring WSNs. In data aggregation a node may pack its input data into a different size outputted, reduce unnecessary data transmission and save energy, which extends the lifetime of network.

For common situations, WSNs are mainly used in a two dimensional environment. Recently there are emerging applications deploying WSNs in a three dimensional environment, such as marine environment monitoring and safety monitoring for coal mine that have attracted interesting attention. Compared to 2D deployment, due to the sufficient increase in the number of sensor nodes within the coverage of WSNs in a 3D deployment, the limit in power storage and supply remains even more severe for WSNs in 3D applications. In this paper, we present a method to maximize network lifetime by balancing the energy consumption at all nodes for 3D WSNs deployment. The main contributions of this paper can be summarized as follows:

- Similar to the zone based routing scheme in [1], we propose a routing scheme called tiled-block routing for 3D environment. Our work is inspired and extends the solution of [1] from $2 \mathrm{D}$ to $3 \mathrm{D}$.

- Using a method similar to corona-partition, we propose a decomposing method that decomposes a 3D monitoring sphere space into sphere-coronas, and then break energy consumption balance down to two levels: intra sphere-corona and inter sphere-corona.

- We propose a scheme to achieve intra energy consumption balance, through partitioning each sphere corona into a set of sphere subcoronas, such that data transmission takes place only between two sphere subcoronas that have the same relative positions in two neighbouring sphere-coronas.

- We propose a scheme to achieve inter energy consumption balance, by computing a proper data distribution ratio for each sphere-corona between direct and hop-by-hop transmission.
- We show that maximizing network lifetime can be achieved through setting an optimal number of sphere-coronas and that of sphere subcoronas within each corona.

This paper is organized as follows: Section 2 presents the system model and the statement of problems. Sections 3 and 4 give the solutions for the intra sphere corona energy balance problem and inter sphere-corona energy balance problem. Section 5 solves the maximizing network lifetime problem. Section 6 presents a protocol called ECBDC and Section 7 give the solutions to extend ECBDC to large-scale sensor network. In Section 8 , the ECBDC is evaluated through simulation and compared to other protocols. And the conclusion of this paper is given in Section 9.

\section{System Models and Problem Statement}

\subsection{Network Model}

We assume that all sensor nodes are distributed in a sphere space $\mathrm{S}$ of radius $\mathrm{R}$, in which the node distribution density is $\rho$. There is only one sink node that is located at the core of S. All nodes have the same maximum range of transmission $T_{\max }$ that is larger than $\mathrm{R}$ and the same amount of initial energy. Every node knows its geographic location.

The data operation is divided into $\mathrm{T}$ rounds. In one word, nodes receive data and generate data then transmit it in one round. In the gap of two adjacent rounds, nodes turn off its radio to save the energy.

\subsection{Data Aggregation Model}

We use a function [25] to illustrate the data aggregation model:

$$
\varphi(x)=m x+c
$$

where $\varphi(x)$ is the amount of data output, $x$ is the amount of data received, $m$ is the aggregation proportion and $\mathrm{c}$ is a const that depends on specific situation:

- If $m=0$ and $c>0$, the amount of data that will be transmitted in one node in one round is small so that it can be put in a single packet.

- If $\mathrm{m}=1$ and $\mathrm{c}=0$, in this case, the node does not perform any data aggregation.

- If $0<\mathrm{m}<1$ and $\mathrm{c}=0$, the node compresses the data output by a factor of $m$.

\subsection{Energy Model}

The energy consumption function is given below:

$$
\varepsilon(x)=\epsilon_{\text {elec }}+\epsilon_{\text {amp }} * x^{k}
$$


where $x^{k}$ is the total energy consumption of transmitting one bit over distance $x, \in_{\text {elec }}$ is the energy consumed by transmitter electronics, and $\epsilon_{a m p}$ is the transmitting amplifier and $k(k \leq 2)$ is the propagation loss exponent.

When the node is receiving data, only the receiving circuit is used, for the energy consumption for receiving one bit by one node is $\varepsilon_{r}=\epsilon_{\text {elec }}$

In this paper, we ignore the energy consumed by generating data for nodes, which is small compared with energy consumption for transmission and reception.

\subsection{Problem Statement}

As shown in Figure 1, the monitoring 3D sphere space is divided into n concentric sphere-coronas $(S C) S C_{1}, S C_{2}$, $S C_{n}$ with the same width $r(r=R / n)$. Similar to $2 \mathrm{D}$ scheme, each node has two ways to transmit its data: directly to the sink and hop-by-hop to a node in the neighbor sphere-corona which is nearer to the sink. Balanced energy consumption can be achieved by allocating data in these two ways.

Definition 1: The data distribution ratio of node $\mathrm{u}$, which is denoted by $p(u)$, is defined as the ratio of the amount of data transmitted in direct mode to the amount of data transmitted in hop-by-hop mode:

$$
P(u)=\frac{D(u)}{F(u)+D(u)}
$$

Let $\mathrm{T}$ be the total number of rounds during the whole network lifetime, and 1 be the number of bits of data generated by one node in one round. $D(u)$ denotes the amount of data transmitted in direct mode in $\mathrm{T}$ rounds, and $F(u)$ denotes the amount of data transmitted in hop-by-hop mode. To solve this problem, we should firstly solve the following sub-problems:

- How to achieve energy consumption balance among nodes within each sphere-corona?

- How to achieve energy consumption balance among

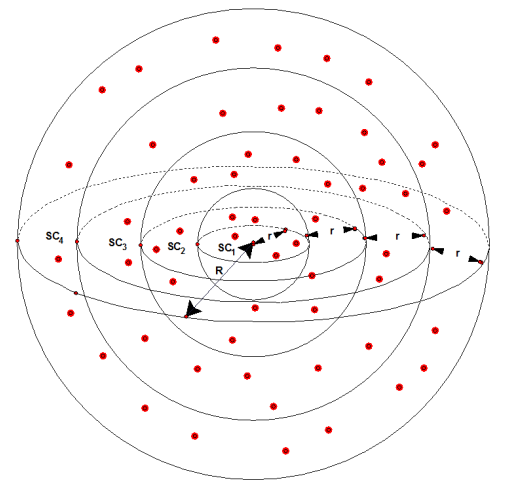

Figure 1. Illustration of network division $(n=4)$. nodes in different sphere-coronas?

- How to get the optimal number of sphere-coronas $n$ in order to maximizing the network lifetime?

We will address these issues in the subsequent sections respectively.

\section{Intra Sphere-Corona Energy Consumption Balancing}

This section focuses on solving the sub-problem of energy consumption balancing (ECB) among nodes within each sphere-corona.

\subsection{Sufficient and Necessary Condition for Intra Sphere-Corona ECB}

Firstly, we give the energy consumption function based on the energy model in Subsection 2.3:

$$
\mathrm{E}(u)=F(u) * \varepsilon_{t}\left(r^{\prime}\right)+D(\mathrm{u}) * \varepsilon_{t}(i r)+S(u) * \varepsilon_{t}
$$

where $\mathrm{E}(u)$ is the total amount of energy consumed by node $\mathrm{u}$ in $\mathrm{T}$ rounds, and $S(u)$ denotes the amount of data received by node $u$ in $T$ rounds. Then $F(u) * \varepsilon_{t}\left(r^{\prime}\right)$ is the amount of energy consumed by node $\mathrm{u}$ when transmitting data in hop-by-hop mode in $\mathrm{T}$ rounds, $D(\mathrm{u}) * \varepsilon_{t}(i r)$ is the amount of energy consumed by node $\mathrm{u}$ when transmitting data in direct mode in $\mathrm{T}$ rounds, and $S(u) * \varepsilon$ is the amount of energy consumed by node $\mathrm{u}$ when receiving data in T rounds.

Lemma 1: [1] $\forall u, v \in C_{i} \quad F(u)=F(v)$ and $D(u)=D(v)$ if and only if $S(u)=S(v)$.

Theorem 1: [1] Energy consumption is balanced among nodes within in $S C_{i}$ if the amount of data received by nodes in $S C_{i}$ is balanced.

Lemma 2:

$$
\frac{s_{i}}{f_{i+1}}=\frac{1+3 i^{2}+3 i}{1+3 i^{2}-3 i} \quad 1 \leq i \leq n
$$

Proof: Let $N_{c_{i}}$ and $N_{c i+1}$ be the number of nodes in $S C_{i}$ and $S C_{i+1}$, thus

$$
\begin{gathered}
N_{c i}=\rho\left[\frac{4}{4} \pi(i r)^{3}-\frac{4}{3} \pi(i-1)^{3} r^{3}\right]=\frac{4}{3} \pi r^{3} \rho\left(1+3 i^{2}-3 i\right) \\
N_{c i+1}=\rho\left[\frac{4}{3} \pi(i+1)^{3} r^{3}-\frac{4}{3} \pi i^{3} r^{3}\right]=\frac{4}{3} \pi r^{3} \rho\left(1+3 i^{2}+3 i\right) \\
\because N_{c_{i}} \cdot s_{i}=N_{c i+1} \cdot f_{i+1}, \\
\therefore \frac{s_{i}}{f_{i+1}}=\frac{1+3 i^{2}+3 i}{1+3 i^{2}-3 i} .
\end{gathered}
$$




\subsection{Tiled-block Based Routing Scheme}

The basic idea of the Tiled-block (TB) is illustrated as follows: As shown in Figure 2, each sphere-corona is divided into $\omega$ sphere subcoronas with equal width $\mathrm{r} / w$, then the spherical surface of each sphere subcorona is divided with latitudes of number $L_{a}$ and then longitudes of number $\mathrm{L}_{O}$, with $L_{a}$ and $\mathrm{L}_{O}$ defined by users. Moreover, the Lo longitudes divide the spherical surface into $\mathrm{L}_{O}$ same cambered surfaces with equal radian and equal width, and $\mathrm{La}$ latitudes divide each longitude into $L_{a}+1$ equilength arcs. Nodes in sphere subcorona $\mathrm{SCi}$ use two tuples to express their Tiled-block ID $(e, g h, k)$. Index $h$ locates nodes in latitudinal direction and index $\kappa$ locates nodes in longitudinal direction.

For any node $u,\left(\alpha_{u}, \beta_{u}, \gamma_{u}\right)$ denotes the three dimensional polar coordinates of $\mathrm{u}$, and $T B_{i, j, h, k}$ denotes the tiled block $(h, k)$ in $S C_{i, j}, h_{i}$ and $h_{i-1}$ denote the distance between plane $A B C D$ and plane $E F G H$ and the distance between plane $I J K L$ and plane $M N O P$ respectively.

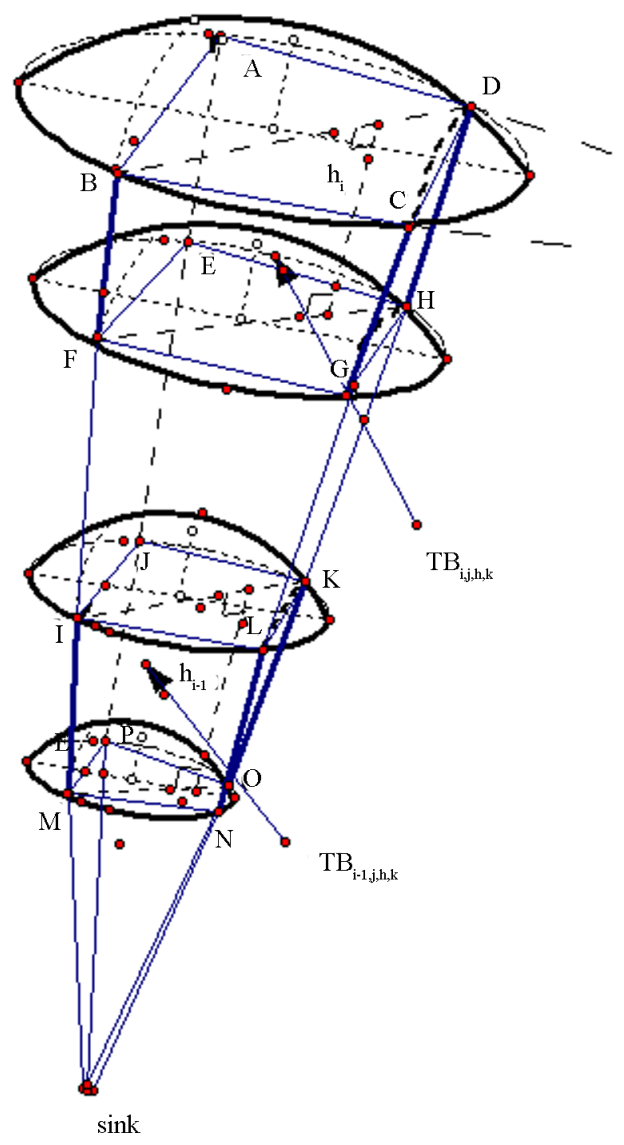

Figure 2. $\mathrm{TB}_{i-1, j, h, k}$ and $\mathrm{TB}_{i, j, h, k}$ in a sphere space.
Lemma 3:

$$
\frac{\overline{B C}}{\overline{I L}}=\frac{\overline{A B}}{\overline{I J}}=\frac{(i-1) r+\frac{j r}{w}}{(i-2) r+\frac{j r}{w}}
$$

Proof: As shown in Figure 2, let $\alpha_{\overline{B C}}$ be the radian of $B C, \mathrm{R}_{B C}$ be the radius of spherical surface which $B C$ belongs to, and $\overline{B C}$ be the length of line whose two endpoints are nodes $B$ and $C$. Obviously, $\overline{A D}=\overline{B C}$, because $A D$ and $B C$ belong to the same spherical surface and the length of arc $A D$ equals to the length of arc $B C$. With the same reason, $\overline{E H}=\overline{F G}$, $\overline{J K}=\overline{I L}, \overline{O P}=\overline{M N}$

$$
\begin{aligned}
& \because \alpha_{\overline{B C}}=\alpha_{\bar{L}}, \alpha_{\overline{A B}}=\alpha_{\overline{I J}} \\
& \overline{B C}=2 \times \sin \frac{\alpha_{\overline{B C}}}{2} \times \mathrm{R}_{\overline{B C}} \\
&=2 \times \sin \frac{\alpha_{\overline{B C}}}{2} \times\left((i-1) r+\frac{j r}{w}\right) \\
& \overline{I L}=2 \times \sin \frac{\alpha_{\overline{I L}}}{2} \times \mathrm{R}_{\overline{I L}} \\
&=2 \times \sin \frac{\alpha_{\overparen{I L}}}{2} \times\left((i-2) r+\frac{j r}{w}\right) \\
& \therefore \frac{\overline{B C}}{\overline{I L}}=\frac{(i-1) r+\frac{j r}{w}}{(i-2) r+\frac{j r}{w}} \\
& \overline{A B}=2 \times \sin \frac{\alpha_{\overline{A B}}}{2} \times \mathrm{R}_{\overline{A B}} \\
&=2 \times \sin \frac{\alpha_{\overline{A B}}}{2} \times\left((i-1) r+\frac{j r}{w}\right) \\
& \overline{I J}=2 \times \sin \frac{\alpha_{I J}}{2} \times \mathrm{R}_{\overline{I J}} \\
&=2 \times \sin \frac{\alpha_{\overline{I J}}}{2} \times\left((i-2) r+\frac{j r}{w}\right) \\
& \therefore \frac{A B}{\overline{I J}}= \frac{(i-1) r+\frac{j r}{w}}{(i-2) r+\frac{j r}{w}}=\frac{\overline{B C}}{\overline{I L}} .
\end{aligned}
$$

Lemma 4

$$
h_{i}=h_{i-1} \quad 2 \leq i \leq n
$$

Proof: As shown in Figure 2,

$$
h_{i}=F S=\cos \angle B F S \times B F=\cos \angle B F S \times \frac{r}{w}
$$




$$
h_{i-1}=M T=\cos \angle I M T \times I M=\cos \angle I M T \times \frac{r}{w}
$$

$\because \angle B F S=\angle I M T$

$\therefore h_{i}=h_{i-1}$, Lemma holds.

Let $V_{T B_{i, j, h, k}}$ and $V_{T B_{i-1, j, h, k}}$ be the volume of $T B_{i, j, h, k}$ and $T B_{i-1, j, h, k}$ respectively. We get lemma 5 below.

Lemma 5

$$
\frac{V_{T B_{i, j, h, k}}}{V_{T B_{i-1, j, h, k}}}=\left[\frac{(i-1) r+\frac{j r}{w}}{(i-2) r+\frac{j r}{w}}\right]^{2}
$$

Proof: Let $\overline{A B C D}-\overline{E F G H}$ and $\overline{I J K L}-\overline{M N O P}$ refer to the polyhedron in Figure 3 and polyhedron Figure 4 , respectively. Let $V_{\overline{A B C D}-\overline{E F G H}}$ and denote the volume of $\overline{A B C D}-\overline{E F G H}$ and $\overline{I J K L}-\overline{M N O P}$ respectively.

$\overline{A^{\prime} B^{\prime} C^{\prime} D^{\prime}}-\overline{E^{\prime} F^{\prime} G^{\prime} H^{\prime}}$ is a polyhedron as shown in Figure 3 that satisfies:

- parallelogram $A^{\prime} B^{\prime} C^{\prime} D^{\prime}$ and parallelogram $E^{\prime} F^{\prime} G^{\prime} H^{\prime}$ are rectangles

- $A^{\prime} B^{\prime}=C^{\prime} D^{\prime}=A B$

- $A^{\prime} D^{\prime}=B^{\prime} C^{\prime}=A D=B C$

- $F^{\prime} E^{\prime}=G^{\prime} H^{\prime}=E F$

- $E^{\prime} H^{\prime}=F^{\prime} G^{\prime}=E H=F G$

- rectangle $A^{\prime} B^{\prime} C^{\prime} D^{\prime} / /$ rectangle $E^{\prime} F^{\prime} G^{\prime} H^{\prime}$

$\overline{I^{\prime} J^{\prime} K^{\prime} L^{\prime}}-\overline{M^{\prime} N^{\prime} O^{\prime} P^{\prime}}$ is a polyhedron as shown in Figure 4 that satisfies:

- parallelogram $\mathrm{A}^{\prime} \mathrm{B}^{\prime} \mathrm{C}^{\prime} \mathrm{D}^{\prime}$ and parallelogram $\mathrm{E}^{\prime} \mathrm{F}^{\prime} \mathrm{G}^{\prime} \mathrm{H}^{\prime}$ are rectangles

- $A^{\prime} B^{\prime}=C^{\prime} D^{\prime}=A B$

- $A^{\prime} D^{\prime}=B^{\prime} C^{\prime}=A D=B C$

- $F^{\prime} E^{\prime}=G^{\prime} H^{\prime}=E F$

- $E^{\prime} H^{\prime}=F^{\prime} G^{\prime}=E H=F G$

- rectangle $A^{\prime} B^{\prime} C^{\prime} D^{\prime} / /$ rectangle $E^{\prime} F^{\prime} G^{\prime} H^{\prime}$

As the volume of $T B_{i, j, h, k}$ and $T B_{i-1, j, h, k}$ can not be computed directly, we use $V_{\overline{A B C D}-\overline{E F G H}}$ and $V_{\overline{I J K L}-\overline{M N O P}}$ to approximatively express $V_{T B_{i, j, h, k}}$ and $V_{T B_{i-1, j, h, k}}$. By lemma 3 and lemma 4 ,

$$
\begin{gathered}
\frac{V_{T B_{i, j}, h, k}}{V_{T B_{i-1, j, h, k}}}=\frac{V_{A B C D-E F G H}}{V} \\
\approx \frac{V_{\overline{A B C D}-\overline{E F G H}}}{V_{\overline{I J K L}-\overline{M N O P}}}
\end{gathered}
$$

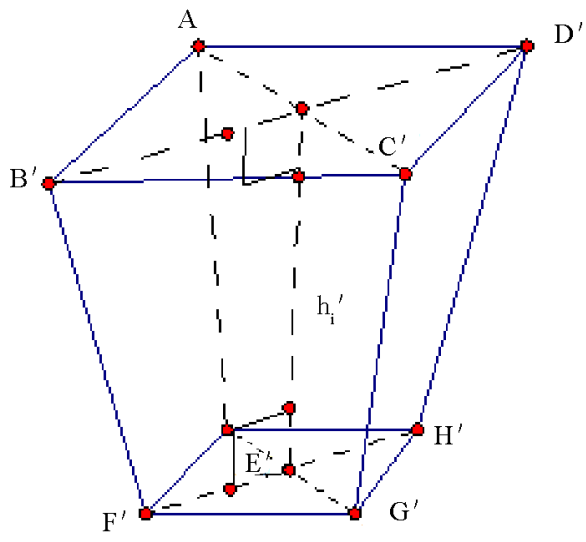

Figure 3 . Polyhedron $\overline{A^{\prime} B^{\prime} C^{\prime} D^{\prime}}-\overline{E^{\prime} F^{\prime} G^{\prime} H^{\prime}}$.

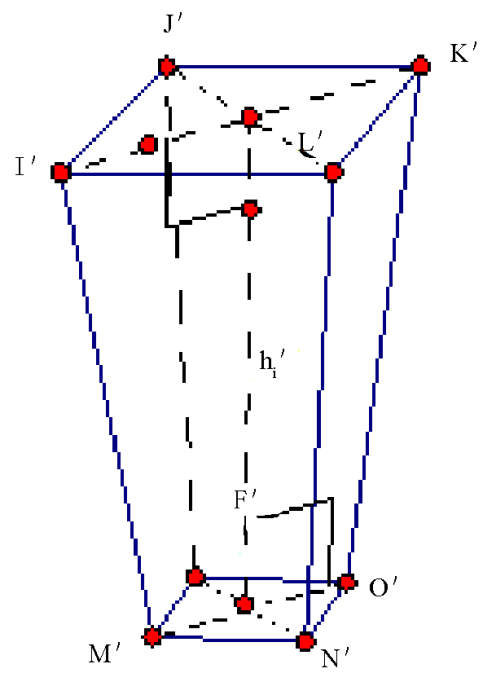

Figure 4. Polyhedron $\overline{I^{\prime} J^{\prime} K^{\prime} L^{\prime}}-\overline{M^{\prime} N^{\prime} O^{\prime} P^{\prime}}$.

$$
\begin{aligned}
& \approx \frac{V_{\overline{A^{\prime} B^{\prime} C^{\prime} D^{\prime}}-\overline{E^{\prime} F^{\prime} G^{\prime} H^{\prime}}}}{V_{\overline{I^{\prime} J^{\prime} K^{\prime} L^{\prime}}-\overline{M^{\prime} N^{\prime} O^{\prime} P^{\prime}}}} . \\
& =\left(\frac{h_{i}}{6}[(\overline{A B} \times \overline{B C})+(\overline{B C}+\overline{F G})(\overline{A B}+\overline{E F})+(\overline{E F} \times \overline{F G})]\right) / \\
& \left(\frac{h_{i-1}}{6}[(\overline{I J} \times \overline{I L})+(\overline{I L}+\overline{M N})(\overline{I J}+\overline{M P})+(\overline{M P} \times \overline{M N})]\right)
\end{aligned}
$$$$
\approx\left[\frac{(i-1) r+\frac{j r}{w}}{(i-2) r+\frac{j r}{w}}\right]^{2}
$$

Lemma 6: For $\forall u, v \in C_{i, j}, S(u)=S(v)$.

Proof: Firstly, for any node $u \in T B_{n-1, j, k, h}$

$$
S(u)=\frac{V_{T B_{n, j, h, k}} \times \rho \times\left(1-p_{n}\right) \times \varphi(T l)}{V_{T B_{n-1, j, h, k}} \times \rho}
$$




$$
=\frac{V_{T B_{n, j, h, k}}}{V_{T B_{n-1, j, h, k}}}\left(1-p_{n}\right) \varphi(T l)
$$

By lemma 5

$$
S(u)=\left[\frac{(n-1) r+\frac{j r}{w}}{(n-2) r+\frac{j r}{w}}\right],
$$

we can see that $S(u)$ is a function of variable $j, p_{n}$, $w$, which are the same to for nodes in $T B_{n-1, j, h, k}$. Besides, $n, T$ and $l$ are constants. Therefore, for any nodes $u, v, S(u)=S(v)$. Secondly, suppose that the amount of data received by any node in $S C_{i}$ is balanced. For any node $u \in T B_{i-1, j, h, k}$,

$$
\begin{aligned}
S(u) & =\frac{V_{T B_{i, j, h, k}} \times \rho \times\left(1-p_{i}\right) \times \varphi\left(T l+S_{i, j}\right)}{V_{T B_{i-1, j, h, k}} \times \rho} \\
& =\frac{V_{T B_{i, j, h, k}}}{V_{T B_{i-1, j, h, k}}}\left(1-p_{i} \varphi\left(T l+S_{i, j}\right)\right) \\
& =\left[\frac{(i-1) r+\frac{j}{r w}}{(i-2) r+\frac{j}{r w}}\right]^{2}\left(1-p_{i} \varphi\left(T l+S_{i, j}\right)\right)
\end{aligned}
$$

So, the amount of data received by nodes in $S C_{i-1, j}$ is balanced.

Theorem 2 The amount of data received by nodes in $S C_{i}$ is balanced in Tiled-block based on routing scheme if

$$
r_{i, j}=r \sqrt[3]{(i-1)^{3}+\frac{1+3 i^{2}-3 i}{1+3 i^{2}+s i}\left(\frac{3 i^{2} j}{w}+\frac{3 i j^{2}}{w^{2}}+\frac{j^{3}}{w^{3}}\right)}
$$

Proof: By lemma 2,

$$
\begin{aligned}
& \frac{V_{C_{i+1,1}}}{V_{C_{i, 1}}}=\frac{V_{C_{i+1,2}}}{V_{C_{i, 2}}}=\cdots=\frac{V_{C_{i+1, w}}}{V_{C_{i, w}}}=\frac{1+3 i^{2}+3 i}{1+3 i_{2}-3 i} \\
& \because \frac{\sum_{k=1}^{j} V_{C_{i+1, k}}}{\sum_{k=1}^{j} V_{C_{i, k}}}=\frac{\frac{4}{3} \pi\left(i r+\frac{j r}{w}\right)^{3}-\frac{4}{3} \pi(i r)^{3}}{\frac{4}{3} \pi r_{i, j}^{3}-\frac{4}{3} \pi(i-1)^{3} r^{3}} \\
& =\frac{1+3 i^{2}+3 i}{1+3 i_{2}-3 i} \\
& \therefore r_{i, j}=r \sqrt[3]{(i-1)^{3}+\frac{1+3 i^{2}-3 i}{1+3 i^{2}+s i}\left(\frac{3 i^{2} j}{w}+\frac{3 i j^{2}}{w^{2}}+\frac{j^{3}}{w^{3}}\right)}
\end{aligned}
$$

\section{Inter Sphere-Corona Energy Consump- tion Balancing}

This section focus on solving the sub-problem of balancing energy consumption among nodes in different sphere-coronas.

\subsection{Hop-by-Hop Transmission Distance}

In this section, we compute the hop-by-hop transmission distance $r_{i}^{\prime}$ for each sphere-corona $S C_{i}$.

Theorem 3: Let $w^{*}$ be the optimal number of sphere subcoronas for maximizing $r_{i}^{\prime}$, then,

$$
\begin{gathered}
r_{i}^{\prime} \approx r\left(\left(1+\frac{1}{w^{*}}\right)^{2}+\left[\frac{(2 i-1) w^{*}-1}{2 i w^{*}}\right]^{2}\right. \\
\left.\left[4 \sin ^{2} \frac{\pi}{2\left(L_{a}+1\right)}+\sin ^{2} \frac{\pi}{L_{o}}\right] i^{2}\right)^{\frac{1}{2}}
\end{gathered}
$$

where $w^{*}=\max _{i=1,2, \cdots n} h(i)$, function $\quad \bar{w}=h(i) \quad$ is determined by equation below:

$$
\begin{aligned}
& \frac{r^{2}}{2}\left[4 \sin ^{2} \frac{\pi}{2\left(L_{a}+1\right)}+\sin ^{2} \frac{\pi}{L_{o}}\right]\left[(2 i-1) \frac{1}{\bar{w}^{2}}-\frac{1}{\bar{w}^{3}}\right] \\
& -2 r^{2}\left(\frac{1}{\bar{w}^{2}}+\frac{1}{\bar{w}^{3}}\right)=0
\end{aligned}
$$

Proof: All nodes in $S C_{i}$ have the same hop-by-hop transmission distance $r_{i}^{\prime}$. We assume that point $Y$ and point $Z$ are two points located in the same sphere subcorona and keep the same distance to sink. Obviously, these two points are located on the same spherical surface centered at the sink. Then let $\alpha_{\curlyvee \mathrm{YZ}}$ be the radian of $Y Z$, and $R_{Y Z}$ be the radius of spherical surface to which $Y Z$ belongs.

As shown in Figure 5, obviously the hop-by-hop transmission distance $r_{i}^{\prime}=\overline{A C^{\prime}}$, now we are going to compute the length of $\overline{A C^{\prime}}$. What shown in Figure 6 is the top view of tiled-block $T B_{i, j, h, k}$, and the profile of arc $B C$ in Figure 7. Point $O$ in (b) denotes the sink, which is the core of the sphere space. We can get,

$$
\begin{aligned}
\overline{D C} & =2 \sin \frac{\alpha_{\overline{D C}}}{2} \times R_{\overline{D C}} \\
& =2 \sin \frac{\pi}{2\left(L_{a}+1\right)} \times \overline{O C} \\
& =2 \sin \frac{\pi}{2\left(L_{a}+1\right)}\left[(i-1) r+\frac{j r}{w}\right]
\end{aligned}
$$




$$
\begin{aligned}
R_{\mathrm{BC}}= & \overline{B N} \\
= & \sin \angle B M O \times \overline{\mathrm{BM}} \\
= & 2 \sin \left(\frac{\pi-\angle B O M}{2}\right) \\
& \times \sin \frac{\angle B M O}{2}[(i-1) r+f j r w] \\
\because \angle B M O= & \frac{p i}{L_{a}+1}(h-1) \\
\therefore R_{\mathrm{BC}}= & 2 \sin \left[f 12\left(\pi-\frac{\pi(h-1)}{L_{a}+1}\right)\right] \sin (f \pi(h-1) \\
& \left.\times 2\left(L_{a}+1\right)\right)\left[(i-1) r+\frac{j r}{w}\right] \\
= & \cos \frac{\pi(h-1)}{2\left(L_{a}+1\right)} \sin \frac{\pi(h-1)}{2\left(L_{a}+1\right)} \times\left[(i-1) r+\frac{j r}{w}\right]
\end{aligned}
$$

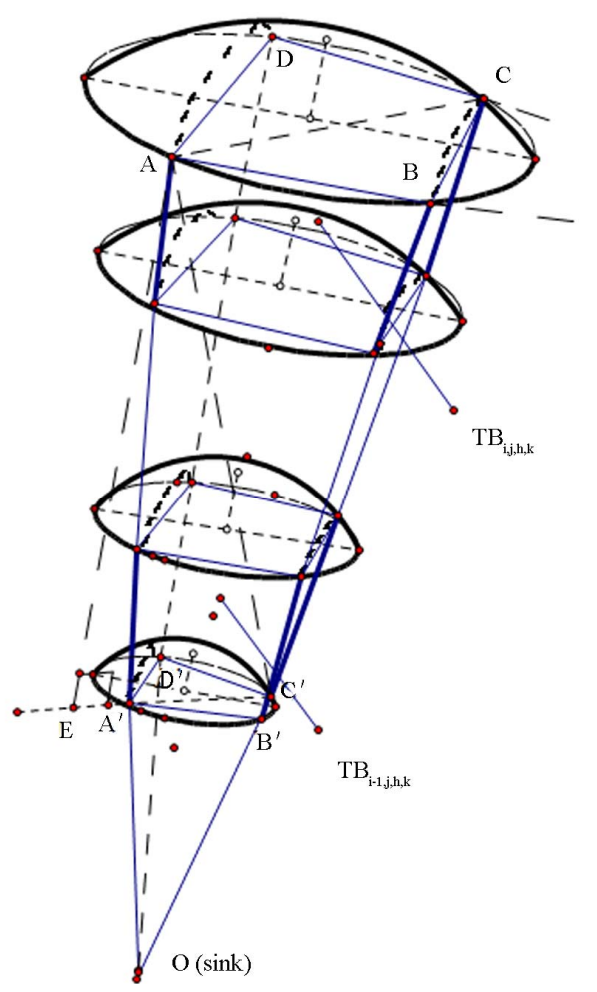

Figure 5. Illustration of the range of $r_{i}^{\prime}$.

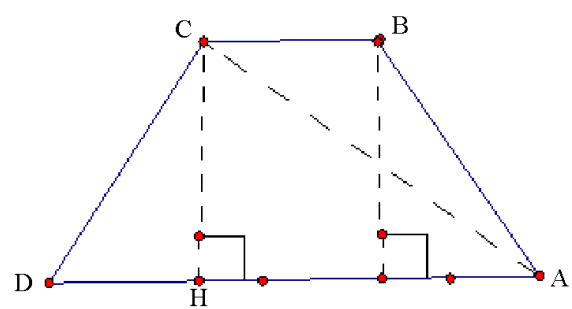

Figure 6. The cross section of $T B_{i, j, h, k}$.
In the same way, we can also get $\overline{A D}$ :

$$
\begin{aligned}
\overline{A D}=2 & \sin \frac{\pi}{L_{o}} \cos \frac{\pi h}{2\left(L_{a}+1\right)} \sin \frac{\pi h}{2\left(L_{a}+1\right)}\left[(i-1) r+\frac{j r}{w}\right] . \\
\therefore \overline{A C}= & \sqrt{\overline{C H}^{2}+\overline{A H}^{2}} \\
= & \sqrt{\overline{D C}^{2}+\overline{A D} \times \overline{B C}} \\
= & \left(\left[2 \sin \frac{\pi}{2\left(L_{a}+1\right)}\left[(i-1) r+\frac{j r}{w}\right]\right)^{2}+\sin ^{2} \frac{\pi}{L_{o}}\right. \\
& \left.\times\left[(i-1) r+\frac{j r}{w}\right]^{2} \sin \frac{\pi h}{\left(L_{a}+1\right)} \sin \frac{\pi(h-1)}{\left(L_{a}+1\right)}\right)^{12} \\
& \quad \frac{\overline{A^{\prime} C^{\prime}}}{\overline{A C}}=\frac{\overline{O A^{\prime}}}{\overline{O A}} \approx \frac{(i-2) r+\frac{(j-1) r}{w}}{(i-1) r+\frac{j r}{w}}, \\
& \quad\left[\frac{(i-2) r+\frac{(j-1) r}{A^{\prime} C^{\prime}}+\overline{A C} \approx\left[\frac{(i-1) r+\frac{j r}{w}}{(2 i-3)}\right] \times \overline{A C}}{(i-1) w+j}\right] \\
& {\left.\left.\left[(i-1) r+\frac{j r}{w}\right]\right)^{2}+\sin ^{2} \frac{\pi}{L_{o}}\left[(i-1) r+\frac{j r}{w}\right]^{2}\right) }
\end{aligned}
$$

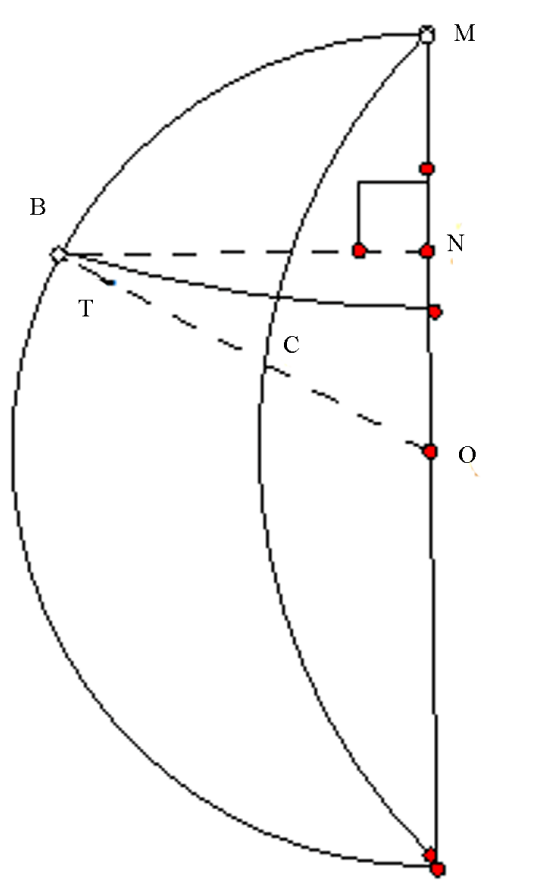

Figure 7. The profile of arc $B C$ 


$$
\begin{aligned}
& \leq \frac{(2 i-1) w-1}{i w} \times\left(\left(2 \sin \frac{\pi}{2\left(L_{a}+1\right)} i r\right)^{2}\right. \\
& \left.+\sin ^{2} \frac{\pi}{L_{o}}(i r)^{2}\right)^{\frac{1}{2}}, \\
& \left(\frac{\overline{A^{\prime} C^{\prime}}+\overline{A C}}{2}\right)^{2}<\left[\frac{(2 i-1) w-1}{2 i w}\right]^{2} \times(i r)^{2} \\
& {\left[\left(2 \sin \left(\frac{\pi}{2\left(L_{a}+1\right)}\right)\right)^{2}\right.} \\
& \left.+\sin ^{2} \frac{\pi}{L_{o}}\right] \text {, } \\
& \overline{A C^{\prime}}=\sqrt{\overline{A E}^{2}+{\overline{E C^{\prime}}}^{2}} \\
& \approx \sqrt{{\overline{A A^{\prime}}}^{2}+{\overline{E C^{\prime}}}^{2}} \\
& =\sqrt{{\overline{A A^{\prime}}}^{2}+\left(\frac{\overline{A C}+\overline{A^{\prime} C^{\prime}}}{2}\right)^{2}} \\
& <\left(\left(r+\frac{r}{w}\right)^{2}+\left[\frac{(2 i-1) w-1}{2 i w}\right]^{2} \times(i r)^{2}\right. \\
& \left.\left[\left(2 \sin \left(\frac{\pi}{2\left(L_{a}+1\right)}\right)\right)^{2}+\sin ^{2} \frac{\pi}{L_{o}}\right]\right)^{\frac{1}{2}} \\
& =r\left(\left(1+\frac{1}{w}\right)^{2}+\left[\frac{(2 i-1) w-1}{2 i w}\right]^{2} \times i^{2}\right. \\
& \left.\left[\left(2 \sin \left(\frac{\pi}{2\left(L_{a}+1\right)}\right)\right)^{2}+\sin ^{2} \frac{\pi}{L_{o}}\right]\right)^{\frac{1}{2}} \\
& \text { Let } \quad g(w)=\left(1+\frac{1}{w}\right)^{2}+\left[\frac{(2 i-1) w-1}{2 i w}\right]^{2} \\
& \times i^{2}\left[\left(2 \sin \left(\frac{\pi}{2\left(L_{a}+1\right)}\right)\right)^{2}+\sin ^{2} \frac{\pi}{L_{o}}\right]
\end{aligned}
$$

Then let $g^{\prime}(w)$ is the derivative of $g(w)$, and $g^{\prime \prime}(w)$ is the second derivative of $g(w)$. When $i>2.5$,

$$
\begin{gathered}
g^{\prime \prime}(w)=\frac{1}{w^{3}}\left(4-(2 i-1)\left[4 \sin ^{2} \frac{\pi}{2\left(L_{a}+1\right)}+\sin ^{2} \frac{\pi}{L_{o}}\right]\right. \\
\left.+\frac{1}{w^{4}}\left(6+\frac{3}{2}\left[4 \sin ^{2} \frac{\pi}{2\left(L_{a}+1\right)}+\sin ^{2} \frac{\pi}{L_{o}}\right]\right)\right)<0,
\end{gathered}
$$

which means that $g(w)$ is a convex function when $i>2.5$. Let

$$
\begin{aligned}
g^{\prime}(w)= & \frac{r^{2}}{2}\left[4 \sin ^{2} \frac{\pi}{2\left(L_{a}+1\right)}+\sin ^{2} \frac{\pi}{L_{o}}\right] \\
& {\left[(2 i-1) \frac{1}{\bar{w}^{2}}-\frac{1}{\bar{w}^{3}}\right]-2 r^{2}\left(\frac{1}{\bar{w}^{2}}+\frac{1}{\bar{w}^{3}}\right)=0 . }
\end{aligned}
$$

Let $\bar{w}=h(i), Q(i)=\max \left|r_{i}^{\prime}\right|$. Therefore we can get,

$$
\begin{array}{r}
Q(i)=r\left(\left(1+\frac{1}{w}\right)^{2}+\left[\frac{(2 i-1) w-1}{2 i w}\right]^{2} * i^{2}\right. \\
\left.\left[\left(2 \sin \left(\frac{\pi}{2\left(L_{a}+1\right)}\right)\right)^{2}+\sin ^{2} \frac{\pi}{L_{o}}\right]\right)^{\frac{1}{2}},
\end{array}
$$

where

$$
w^{*}=\max _{i=1,2, \cdots n} h(i) .
$$

We can see that, $r_{i}^{\prime}$ is the linear function of sphere-corona id $i$. Therefore, the hop-by-hop transmission distances for all nodes in $S C_{i}$ are the same, but for nodes in different sphere-coronas may be different.

\subsection{Linear Network Model}

By Lemma 1 and Theorem 1, when energy consumption is balanced among nodes in $S C_{i}$, all nodes in $S C_{i}$ transmit the same amount of data $F_{i}$ in the hop-by-hop way, transmit the same amount of data $D_{i}$ in the direct way, and receive the same amount of data $S_{i}$ from nodes in $C_{i+1}$. Let $f_{i}=\frac{F_{i}}{T}, d_{i}=\frac{D_{i}}{T}$ and $s_{i}=\frac{S_{i}}{T}$. The network can be mapped into a linear model, as shown in Figure 8 based on (1),

$$
f_{i}+d_{i}=m\left(l+s_{i}\right)+c
$$

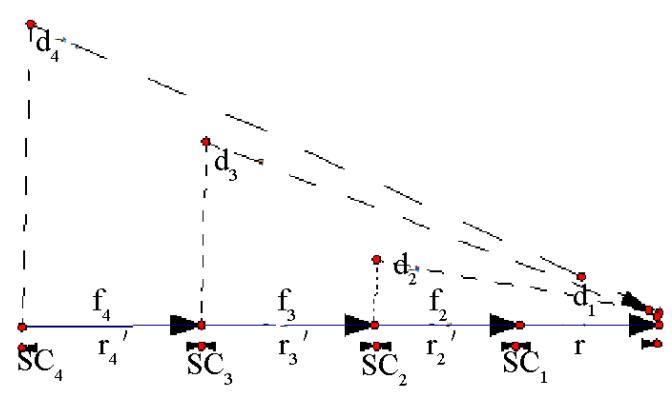

Figure 8. Mapping the network onto a linear model. 
By (3),

$$
p_{i}=\frac{D_{i}}{F_{i}+D_{i}}=\frac{d_{i}}{f_{i}+d_{i}}=\frac{d_{i}}{m\left(f_{i}+d_{i}\right)+c}
$$

Let $e_{i}=\frac{E_{i}}{T}$. By (4),

$$
e_{i}=f_{i} \times \varepsilon_{t}\left(r_{i}^{\prime}\right)+d_{i} \times \varepsilon_{t}(i r)+s_{i} \times \varepsilon_{\text {elec }}
$$

So, the Inter sphere-corona energy consumption balancing problem can be formulated as the following problem of distribution of data transmission:

$$
\left\{\begin{array}{l}
\text { Compute } p_{i} \quad 1 \leq i \leq n \\
\text { s.t. } e_{1}=e_{2}=\cdots=e_{i}=\cdots=e_{n-1}=e_{n} .
\end{array}\right.
$$

\subsection{Optimal Data Distribution Ratio Allocation}

Since energy consumption balance may be achived by optimally distributing the amount of data in direct transmission and hop-by-hop transmission, we focus on computing the optimal data distrubition ratio for each sphere-corona.

Lemma 7:

$$
s_{i}=\left\{\begin{array}{lc}
0 & i=n ; \\
\left(m \times\left(s_{i+1}+l\right)+c-d_{i+1}\right) \times \frac{1+3 i^{2}+3 i}{1+3 i^{2}-3 i} & 1 \leq i \leq n .
\end{array}\right.
$$

Proof: If $i=n, S C_{n}$ is the outermost spherecorona. Since all nodes in $S C_{n}$ do not receive any data, $S_{n}=0$. If $1 \leq i<n$, by Lemma 2 ,

$$
s_{i}=f_{i+1} \frac{1+3 i^{2}+3 i}{1+3 i^{2}-3 i}
$$

By (5), $f_{i}=m\left(s_{i}+l\right)+c-d_{i}$. Therefore,

$$
s_{i}=\left(m \times\left(s_{i+1}+l\right)+c-d_{i+1}\right) \times \frac{1+3 i^{2}+3 i}{1+3 i^{2}-3 i} .
$$

Lemma 8:

$$
\begin{aligned}
d_{i}= & \frac{1}{\varepsilon_{t}(i r)-\varepsilon_{t}\left(r_{i}^{\prime}\right)} \times\left(d_{i+1}\left[\varepsilon_{t}((i+1) r)-\varepsilon_{t}\left(r_{i+1}^{\prime}\right)\right]\right. \\
& +\left[m\left(s_{i+1}+l\right)+c\right] \varepsilon_{t}\left(r_{i+1}{ }^{\prime}\right)-\left(s_{i}-s_{i+1}\right) \times \varepsilon_{\text {elec }} \\
& \left.-\left[m\left(s_{i+1}+l\right)+c\right] \varepsilon_{t}\left(r_{i}^{\prime}\right)\right)
\end{aligned}
$$

where $2 \leq i \leq n$.

Proof: When $i>1$, all nodes have two ways to transmit data: in hop-by-hop way and in direct way. Energy consumption balance can be achieved only when $e_{i}=e_{i+1}$. Thus

$$
\begin{aligned}
& f_{i+1} \times \varepsilon_{t}\left(r_{i+1}^{\prime}\right)+d_{i+1} \times \varepsilon_{t}((i+1) r)+s_{i+1} \times \varepsilon_{\text {elec }} \\
& =f_{i} \times \varepsilon_{t}\left(r_{i}^{\prime}\right)+d_{i} \times \varepsilon_{t}(i r)+s_{i} \times \varepsilon_{\text {elec }} \\
& \therefore \quad f_{i} \times \varepsilon_{t}\left(r_{i}^{\prime}\right)-f_{i+1} \times \varepsilon_{t}\left(r_{i+1}^{\prime}\right)+\left(s_{i}-s_{i+1}\right) \times \varepsilon_{\text {elec }}
\end{aligned}
$$

$$
=d_{i+1} \times \varepsilon_{t}((i+1) r)-d_{i} \times \varepsilon_{t}(i r)
$$

By (5),

$$
\begin{aligned}
d_{i} & =\frac{1}{\varepsilon_{t}(i r)-\varepsilon_{t}\left(r_{i}^{\prime}\right)} \times\left(d_{i+1}\left[\varepsilon((i+1) r)-\varepsilon\left(r_{i+1}^{\prime}\right)\right]\right. \\
& +\left[m\left(s_{i+1}+l\right)+c\right] \varepsilon\left(r_{i+1}^{\prime}\right)-\left(s_{i}-s_{i+1}\right) \times \varepsilon_{\text {elec }} \\
& \left.-\left[m\left(s_{i+1}+l\right)+c\right] \varepsilon_{t}\left(r_{i}^{\prime}\right)\right)
\end{aligned}
$$

Lemma 9

$$
\begin{aligned}
d_{2}= & \frac{1}{\varepsilon_{t}(2 r)-\varepsilon_{t}\left(r_{2}^{\prime}\right)}\left(\left(s_{1}-s_{2}\right) \times \varepsilon_{\text {elec }}+m\left(s_{1} \varepsilon_{t}(r)\right.\right. \\
& \left.\left.-s_{2} \varepsilon_{t}\left(r_{2}^{\prime}\right)\right)+(m l+c)\left(\varepsilon_{t}(r)-\varepsilon_{t}\left(r_{2}^{\prime}\right)\right)\right)
\end{aligned}
$$

Proof: Since all nodes in $S C_{1}$ transmit their data only in direct way, therefore,

$$
\begin{aligned}
e_{1} & =d_{1} \times \varepsilon_{t}(r)+s_{1} \times \varepsilon_{\text {elec }} \\
& =\left[m\left(s_{1}+l\right)+c\right] \times \varepsilon_{t}(r)+s_{1} \times \varepsilon_{\text {elec }}
\end{aligned}
$$

By $e_{1}=e_{2}$,

$$
\begin{aligned}
& {\left[m\left(s_{1}+l\right)+c\right] \times \varepsilon_{t}(r)+s_{1} \times \varepsilon_{\text {elec }} } \\
= & f_{2} \times \varepsilon_{t}\left(r_{2}{ }^{\prime}\right)+d_{2} \times \varepsilon_{t}(2 r)+s_{2} \times \varepsilon_{\text {elec }}
\end{aligned}
$$

Therefore, the lemma holds.

From Lemma 7, $s_{i}$ can be expressed by $s_{i+1}$ and $d_{i+1}$, from Lemma $8, d_{i}$ can be expressed by $r_{i}^{\prime}, s_{i}$, $s_{i+1}$ and $d_{i+1}$, from Lemma $9, d_{2}$ can be expressed by $r_{2}{ }^{\prime}, s_{1}$ and $s_{2}$. for all nodes in $S C_{n}, s_{n}=1$. Therefore, we can compute $d_{i}$ and $f_{i}$ for nodes in each $S C_{i}$ after a few iterations. The detailed algorithm is given below:

Algorithm 1:

1) For $i=1$ to $\mathrm{ndo}$

2) Compute $r_{i}^{\prime}$

3) For $i=n-1$ down to 2 do

4) Express $s_{i}$ by $d_{n}$

5) Express $d_{i}$ by $d_{n}$

6) Express $d_{2}$ by $d_{n}$

7) Compute $d_{n}$ by making $d_{2}$ in Lemma 8 equal to $d_{2}$ in Lemma 9

8) For $i=2$ to $n$ do

9) Compute $d_{i}$ and $s_{1}$

10) $p_{i}=\frac{d_{i}}{m\left(s_{i}+l\right)+c}$

We can see that, the complexity of this algorithm is $O(n)$, where $n$ is the number of sphere-coronas. 


\subsection{Numerical Results and Analysis}

We have done numerical analysis, in order to understand how data compression ratio $m$ influences the data transmission and reception. The system parameters are set as follows:

$$
R=100 \quad, \quad r=10 \quad, \quad n=10 \quad, \quad \varepsilon_{\text {elec }}=50 \mathrm{~nJ} / \mathrm{bit},
$$$$
\varepsilon_{\text {amp }}=100 \mathrm{pJ} / \text { bit , and } k=2 \text {. }
$$

Figure 9 plots the amount of data received per node with different sphere-coronas. We can see that, sensors near the sink node receive more data from hop-by-hop transmission than those far from the sink node. Especially, nodes in the outmost sphere-corona $(\mathrm{id}=10)$ receive no data as no one transmits data to them. This showns our scheme results in a desirable data distribution to achieve energy consumption balance. And we can also see that, as the data compression ratio $\mathrm{m}$ sets smaller, the amount of data received per node in the network becomes also smaller. This phenomenon manifests that data aggregation can reduce data transmitted and hence save energy consumption for WSNs.

Figure 10 plots the ratios of data distribution in different sphere-coronas. The data distribution ratio firstly decreases rapidly, then remains constant more or less in the middle sphere-coronas and finally increases in the last few sphere-coronas. This can be explained as that, energy consumption is proportional to the production of data volume and square of the transmission distance. To maintain a balanced energy consumption, nodes at the two ends have a higher distribution ratio because at the near end nodes have heavy relay burden, but at the far end it just goes to the opposite: long transmitting distance but small data volume. And in Figure 10, it is also easily see that data compression ratio(m) has great influence on the distribution ratios. When $\mathrm{m}$ decreases, data distribution ratio also decreases at a rate following lemmas 5-7.

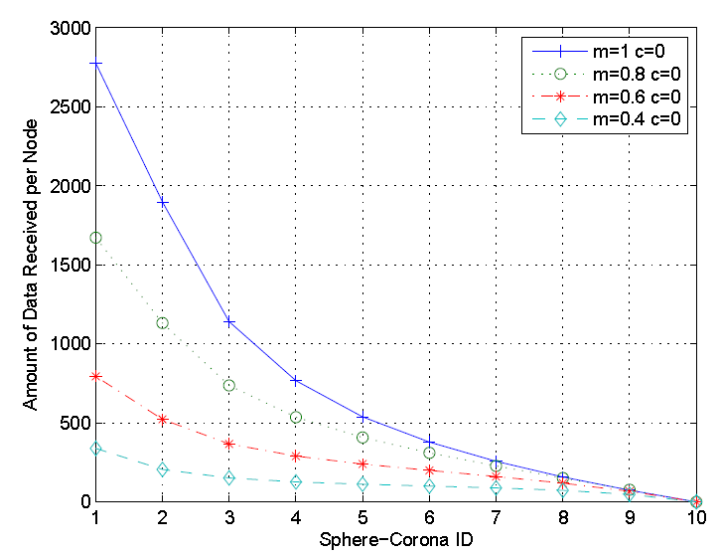

Figure 9. Amount of data received per node for different sphere-coronas with different data aggregation ratios.

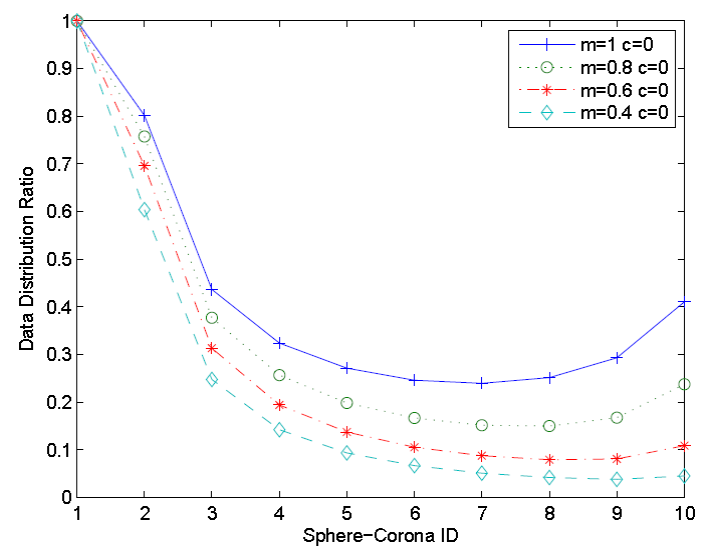

Figure 10. The data distribution ratio different spherecoronas with different data aggregation parameters.

\section{Network Lifetime Maximization}

This section focused on solving the problem of maximizing network lifetime by achieving energy consumption balance among all nodes in the network. For nodes in the outmost sphere-corona $S C_{n}$,

$$
\begin{aligned}
& e_{n}=f_{n} * \varepsilon_{t}\left(r_{n}^{\prime}\right)+d_{n} \times \varepsilon_{t}(n r) \\
& =\left[m l+c-d_{n}\right]\left(\varepsilon_{\text {elec }}+\varepsilon_{\text {amp }}\left(r_{n}^{\prime}\right)^{k}\right) \\
& +d_{n}\left(\varepsilon_{\text {elec }}+\varepsilon_{\text {amp }} R^{k}\right)
\end{aligned}
$$

When energy consumption balance is achieved, all nodes have the same energy consumption equalength to $e_{n}$. Thus, network lifetime maximization can be formulated as the following optimization problem:

$$
\left\{\begin{array}{c}
\min e_{n} ; \\
\text { s.t. } e_{i}=e_{j}, \quad 1 \leq i, j \leq n \\
n r=R .
\end{array}\right.
$$

\subsection{Optimal Number of Sphere Subcoronas}

Given $n$ sphere-coronas, $p_{i}$ and $d_{i}$ can be computed step by step using the algorithm above. By Theorem 3, the optimal number of sphere subcoronas $\mathrm{w}^{*}$ can be obtained from:

$$
w^{*}=\max _{i=1,2, \cdots, n} h(i)
$$

Where function $\bar{w}=h(i)$ is determined by equation below:

$$
\begin{gathered}
\frac{r^{2}}{2}\left[4 \sin ^{2} \frac{\pi}{2\left(L_{a}+1\right)}+\sin ^{2} \frac{\pi}{L_{o}}\right]\left[(2 i-1) \frac{1}{\bar{w}^{2}}-\frac{1}{\bar{w}^{3}}\right] \\
-2 r^{2}\left(\frac{1}{\bar{w}^{2}}+\frac{1}{\bar{w}^{3}}\right)=0
\end{gathered}
$$




\subsection{Optimal Number of Sphere-coronas}

By Lemma 8, $d_{i}$ is a nonlinear function of $r_{i}^{\prime}$. Therefore, getting the optimal number of sphere-coronas is a nonlinear integer programming problem which is NP hard. We can use heuristic algorithm $\mathrm{s}$ to solve this problem.

\section{The ECBDC Protocol}

In this section, a protocol called ECBDC (energy consumption balanced data collecting protocol) is designed. The operation of this protocol is divided into two phases: network set-up phase and data gathering phase.

\subsection{Network Set-up Phase}

As discussed before, network parameters such as the optimal number of sphere-coronas $n$ and the optimal data distribution ratio $p_{i}$ can be computed offline. Therefore, in the set-up phase, the sink broadcasts these parameters to all nodes and then each node establishes its sphere-corona id, source sphere subcorona id, destination sphere-corona id, tiled-block id and hop-by-hop transmission distance $r_{i}^{\prime}$.

Consider three consecutive sphere-coronas $S C_{i-1}$, $S C_{i}$ and $S C_{i+1} . S C_{i}$ acts as destination sphere-corona when the nodes in $S C_{i}$ receive data from nodes in $S C_{i+1}$, and $S C_{i}$ acts as source sphere-corona when nodes in $S C_{i}$ forward data to nodes in $S C_{i-1} . T B_{i, j, h, k}$ is the tiled block in which nodes located.

For any node $u$, let $\left(\alpha_{u}, \beta_{u}, \gamma_{u}\right)$ be the three dimensional polar coordinates of node $u$. Since the network of monitoring sphere space $S$ is divided into $n$ sphere-coronas with equal width $R / n$, we can get the sphere-corona ID $i$ as follows:

$$
i=\left\lceil\frac{r_{u}}{R / n}\right\rceil=\left\lceil\frac{r_{u}^{*} n}{R}\right\rceil
$$

When node $u$ in $S C_{i}$ forwards their data to the corresponding nodes in $S C_{i-1}, S C_{i}$ acts the source sphere-corona. Since the source sphere-corona is divided into $w$ sphere subcoronas with equal width $(R / n) / w$, the source sphere subcorona ID of node $u$ can be given bellow:

$$
\begin{aligned}
& S_{j}=\left\lceil\frac{r_{u}-(i-1) R / n}{R /(n w)}\right\rceil \\
& =\left\lceil\frac{n w r_{u}-(i-1) R W}{R}\right\rceil
\end{aligned}
$$

When node $u$ in $S C_{i}$ receives data from the corresponding nodes in $S C_{i+1}, S C_{i}$ acts as the destination sphere-corona. By Theorem 2, the destination sphere-corona is divided into $w$ sphere subcoronas with unequal width, so we can not simply get the destination sphere-corona ID like before. We give a simple algorithm based on Theorem 2 to compute the destination sphere-corona ID as follows:

Algorithm 2:

1) For $j=1$ to $w$ do

2) If $r_{u} \leq r_{i, j}$ and $r \geq r_{i, j-1}$

3) Return $j$

4) End for

As illustrated in Subsection 3.2, in Tiled-block based routing scheme, the spherical surface is divided with latitudes of number $L_{a}$ and then longitudes of number $L_{o}$. Moreover, the $L_{o}$ longitudes divide spherical surface into Lo cambered surface with equal radian, and La latitudes divide each longitude into $L_{a}+1$ equilength arcs. Nodes use two-tuples to express their Tiled-block ID (e.g $h, k)$. Tuple $h$ locates nodes in the latitudinal direction and Tuple $k$ locates nodes in the longitudinal direction. For any node $u,\left(\alpha_{u}, \beta_{u}, \gamma_{u}\right)$ denotes the three dimensional polar coordinates of $u$, Therefore,

$$
\begin{aligned}
h & =\left\lceil\frac{\alpha_{u}}{2 \pi / L_{o}}\right\rceil \\
& =\left\lceil\frac{\alpha_{u} L_{o}}{2 \pi}\right\rceil \\
k & =\left\lceil\frac{\beta_{u}}{\pi /\left(L_{a}+1\right)}\right\rceil \\
& =\left\lceil\frac{\alpha_{u}\left(L_{a}+1\right)}{\pi}\right\rceil
\end{aligned}
$$

\subsection{Data Gathering Stage}

As in [1], in ECBDC, all sensor nodes work between two states: active and sleep. In the former state, each node generates data, compresses data and transmits data. In the latter state, each node turns off its radio to save energy.

Let $T_{\text {round }}$ be the time duration for completing one round of data gathering. $T_{\text {round }}$ is divided into $w^{*} n$ time slots $\tau_{0}, \tau_{1}, \cdots, \tau_{w^{*} n-1}$. In time slot $\tau_{i}$, nodes in $S C_{n-\left\lfloor\frac{i}{w}\right\rfloor, w-\left(i-\left\lfloor\frac{i}{w}\right\rfloor_{w}\right)}$ and $S C_{n-\left\lfloor\frac{i}{w}\right\rfloor-1, w-\left(i-\left\lfloor\frac{i}{w}\right\rfloor_{w}\right)}$ wake up, then nodes in $S C_{n-\left\lfloor\frac{i}{w}\right\rfloor, w-\left(i-\left\lfloor\frac{i}{w}\right\rfloor_{w}\right)}$ transmit data to nodes 
in $S C_{n-\left\lfloor\frac{i}{w}\right\rfloor-1, w-\left(i-\left\lfloor\frac{i}{w}\right\rfloor w\right)}$, and in the meantime, other nodes in the sphere space get into sleep state. Let $T_{s}(u)$ and $T_{d}(u)$ be the timers to control node $u$ enter into active state to transmit data and to receive data respectively. Let $l_{i}$ be the length of time slot $\tau_{i}$ which is set as follows:

$$
l_{i}=\frac{V_{S C}-\left\lfloor\frac{i}{w}\right\rfloor, w-\left(i-\left\lfloor\frac{i}{w}\right\rfloor w\right)}{4 / 3 \pi R^{3}},
$$

where $V_{S C}-\left\lfloor\frac{i}{w}\right\rfloor, w-\left(i-\left\lfloor\frac{i}{w}\right\rfloor w\right)$ is the space volume of

$S_{n-\left\lfloor\frac{i}{w}\right\rfloor, w-\left(i-\left\lfloor\frac{i}{w}\right\rfloor w\right)}$.

The data gathering operation follows the DG algorithm proposed in [1], as shown in Appendix A.

\section{Extension to Large-scale Data-gathering Sensor Networks}

In this section, we extend the ECBDC protocol to large-scale WSNs. In ECBDC, one of the preconditions is that the transmission range of each node is not less than the radius of the monitoring sphere space, so that every node can transmit data directly to the sink node when in direct mode. However, practically, most sensor nodes have a limited transmission range and may not transmit data to the sink node directly. Same as [1], we solve this problem with clustering technique [26-32].

We divide the whole network into small clusters. Each cluster is equiped with a special node called cluster head, which is located in the core of the cluster. All nodes in one cluster transmit data to its cluster head directly or hop by hop, then the head node transmit data to the sink node. The cluster head has battery energy which is much more than others. The data gathering in the extended ECBDC is implemented as follows:

- Intra-Cluster: In each cluster, all nodes transmit their data to the cluster head using ECBDC.

- Inter-Cluster: All the cluster heads form a super-cluster, and transmit data to the sink node using ECBDC.

\section{Simulation Results and Analysis}

In this section, simulations have been made to evaluate the performance of ECBDC. As so far there is no known scheme for 3D WSNs, for comparison purpose, we choose two conventional 2D schemes: multihop routing scheme and direct transmitting scheme, and apply them to the $3 \mathrm{D}$ model.

\subsection{Simulation Setup}

Sensor nodes are deployed in a sphere space, and the sink node is located in the core of the sphere. The radius of the sphere varys from $100 \mathrm{~m}$ to $1000 \mathrm{~m}$ and the data compression ratio varys from 0.1 to 1.0 . All sensor nodes have the same initial energy $1 \mathrm{~J}$. In every round, each node generates 100 bits data. For the radio model, $\varepsilon_{\text {elec }}=50 \mathrm{~nJ} / \mathrm{bits}, \varepsilon_{\text {amp }}=100 \mathrm{pJ} / \mathrm{bit} / \mathrm{m}^{2}$, and $k=2$. The value of all parameters are shown in Table 1 below.

\subsection{Comparison with Direct Transmission and Multihop Routing Schemes}

In ECBDC, our target is to balance energy consumption and maximize network lifetime. In the best case, all nodes run out their energy in the same moment. In simulations, we pick up two conventional 2D schemes for comparison purpose: direct transmssion and multihop routing. In the direct method, all nodes in monitoring space transmit their data directly to sink node rather than getting through relay nodes; in the multihop method, every node forwards all its data to the next hop node. The ECBDC protocol and this two 2D schemes all run in the $3 \mathrm{D}$ model proposed in this paper.

Figure 11 plots network lifetime achieved by the three schemes with different radius of the sphere space when the number of sphere-coronas $n=10$ and data compression ratio $m=0.8$. We can see, with the growing $R$, network lifetime decreases dramatically. When $R$ varys from $100 \mathrm{~m}$ to $600 \mathrm{~m}$, ECBDC significantly outperforms other two schemes. And when $R$ varys from $700 \mathrm{~m}$ to $1000 \mathrm{~m}$, though still ECBDC outmatches the rivals, the differences among the three are not great.

Figure 12 plots network lifetime obtained by the three schemes with different ratios of data compression when number of sphere-coronas $n=10$ and sphere space radius $R=100 \mathrm{~m}$. When $m$ varys from 0.1 to 0.3 , the multihop scheme is as good as ECBDC, which manifests that data compression is important, especially for multihop scheme and ECBDC. Effective data compression methods can bring low data compression ratio, reduce the amount of data transmitted, and hence lower energy consumption, prolonging network lifetime. In the figure, it's worth noting that, when $m$ vary from

Table 1. Value of all parameters.

\begin{tabular}{cc}
\hline Parameter & Value \\
\hline Network sphere radius(R) & $100 \mathrm{~m} \square 1000 \mathrm{~m}$ \\
Node deployment density $(\rho)$ & 0.1 nodes $/ \mathrm{m}^{3}$ \\
Initial energy & 1 Joule $/$ node \\
Data generation rate(1) & $100 \mathrm{bits} /$ node $/$ round \\
Data Compression ratio(m) & $0 \square 1$ \\
$\varepsilon_{\text {elec }}$ & $50 \mathrm{~nJ} / \mathrm{bit}$ \\
$\varepsilon_{\text {amp }}$ & $100 \mathrm{pJ} / \mathrm{bit} / \mathrm{m}^{3}$ \\
$\mathrm{k}$ & 2 \\
La & 100 \\
Lo & 100 \\
\hline
\end{tabular}




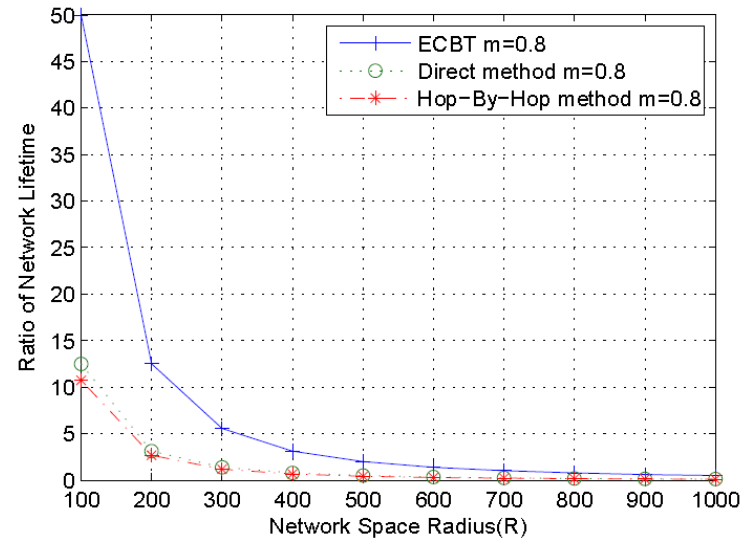

Figure 11. Network lifetime of different routing schemes when $\mathrm{m}=\mathbf{0 . 8}$ and $\mathrm{n}=\mathbf{1 0}$.

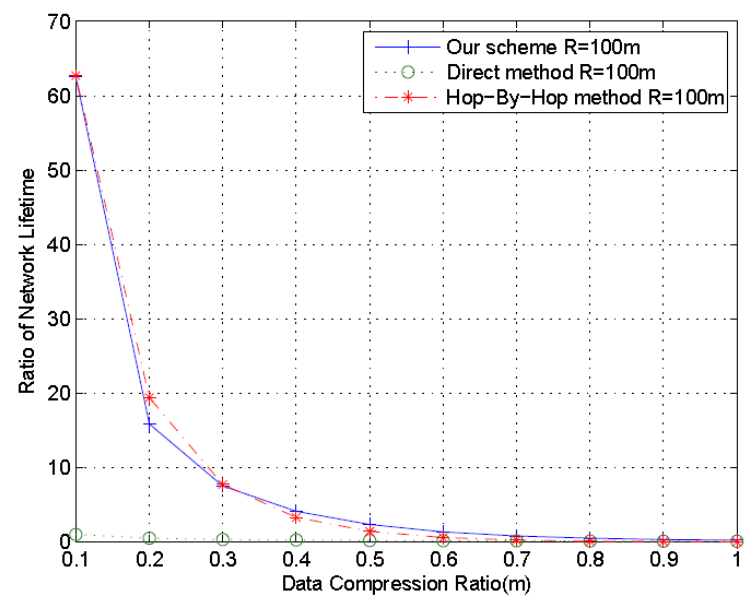

Figure 12. Network lifetime with different routing schemes and data compression ratio when $R=100$ and $n=10$.

0.4 to 1.0 , ECBDC outmatches the rivals. Especially, in the figure, for all value of $m$, the multihop scheme appears better than direct scheme when the space radius $R=100 \mathrm{~m}$, which indicates that, comparing to direct transmission to sink, hop-by-hop transmission is a better choice.

\section{Conclusion}

Unbalanced energy consumption is an important problem in wireless sensor networks, which can dramatically reduce network lifetime. In this paper, we proposed a solution to maximize network lifetime through balancing energy consumption among all nodes in data-gathering sensor networks. We formulated the balancing energy consumption problem as the problem of optimal allocation of transmitting data by combining the ideas of sphere-corona based network partition and mixed-routing strategy together with data aggregation. We presented the solutions for balancing energy consumption among nodes both in the same sphere-coronas and different sphere-coronas. Based on our model, an ECBDC protocol and its extension to large-scale data-gathering sensor networks were developed. Simulation results show that ECBDC can greatly extend network lifetime compared with a multihop transmission scheme and a direct transmission scheme.

\section{References}

[1] H. Zhang and H. Shen, "Balancing Energy Consumption to Maximize Network Lifetime in Data-Gathering Sensor Networks," IEEE Transactions on Parallel and Distributed Systems, Vol. 20, No. 10, 2009, pp. 1526-1539.

[2] I. F. Akyildiz, W. Su, Y. Sankarasubramaniam and E. Cayirci, "A Survey on Sensor Networks," IEEE Communications Magazine, August 2002, pp. 102-114.

[3] A. Brayner and R. Menezes, "Balancing Energy Consumption and Memory Usage in Sensor Data Processing," Proceedings of the 2007 ACM Symposium on Applied Computing, Seoul, 11-15 March 2007.

[4] Y. Xu, J. Heidemann and D. Estrin, "GeographyInformed Energy Conservation for Ad Hoc Routing," Proceedings of the 7th Annual International Conference on Mobile Computing and Networking, Rome, July 2001, pp. 70-84.

[5] D. J. Baker and A. Ephremides, "The Architectural Organization of a Mobile Radio Network via a Distributed Algorithm," IEEE Transactions on Communications, Vol. 29, No. 11, 1981, pp. 1694 -1701.

[6] A. Ephremides, J. E. Wieselthier and D. J. Baker, “A Design Concept for Reliable Mobile Radio Networks with Frequency Hopping Signaling," Proceedings of the IEEE, Vol. 75, No. 1, 1987, pp. 56-73.

[7] M. Ettus, "System Capacity, Latency, and Power Consumption in Multihoprouted SS-CDMA Wireless Networks," Proceedings of IEEE Radio and Wireless Conference, Springs, 9-12 August 1998, pp. 55-58.

[8] R. G. Gallager, P. A. Humblet and P. M. Spira, “A Distributed Algorithm for Minimum Weight Spanning Trees," Massachusetts Institute of Technology, 1979.

[9] T. H. Meng and V. Rodoplu, "Distributed Network Protocols for Wireless Communication," Proceedings of the 1998 IEEE International Symposium on Circuits and Systems, Monterey, Vol. 4, June 1998, pp. IV-600-IV603.

[10] V. Rodoplu and T. H. Meng, "Minimum Energy Mobile Wireless Networks," IEEE Journal on Selected Areas in Communications, Vol. 17, No. 8, 1999, pp. 1333-1344.

[11] T. Shepard, "Decentralized Channel Management in Scalable Multihop Spread Spectrum Packet Radio Networks," Massachusetts Institute of Technology,1995.

[12] S. Singh, M. Woo and C. S. Raghavendra, "PowerAware Routing in Mobile Ad Hoc Networks," Proceedings of Fourth Annual ACM/IEEE International Con- 
ference on Mobile Computing and Networking, Dallas, October 1998, pp. 181-190.

[13] I. Akyildiz, W. Su, Y. Sankarasubramaniam and E. Cyirci, "Wireless Sensor Networks: A Survey," Computer Networks, Vol. 38, No. 4, 2002, pp. 393-422.

[14] F. Zhao and L. Guibas, "Wireless Sensor Networks: An Information Processing Approach," Morgan Kaufmann Publishers, Massachusetts, 2004.

[15] C. Efthymiou, S. Nikoletseas and J. Rolim, "Energy Balanced Data Propagation in Wireless Sensor Networks," Wireless Networks, Vol. 12, No. 6, 2006, pp. 691-707.

[16] W. Guo, Z. Liu and G. Wu, "An Energy-Balanced Transmission Scheme for Sensor Networks," Proceedings of the First International Conference Embedded Networked Sensor Systems, Los Angeles, 5-7 November 2003, pp. 300-301.

[17] O. Powell, P. Leone and J. Rolim, "Energy Optimal Data Propagation in Wireless Sensor Networks," Journal of Parallel and Distributed Computing, Vol. 67, No. 3, 2007, pp. 302-317.

[18] H. Zhang, H. Shen and Y. Tan, "Optimal Energy Balanced Data Gathering in Wireless Sensor Networks," Proceedings of the 21st International Parallel and Distributed Processing Symposium, Long Beach, 26-30 March 2007, pp. 1-10.

[19] A. Zhao, J. Yu and Z. Li, "A Data Aggregation Scheme in Wireless Sensor Networks for Structure Monitoring," Proceedings of the 2009 International Conference on Information Management, Innovation Management and Industrial Engineering, Xi'an, 26-27 December 2009, Vol. 4, pp. 623-626.

[20] J. N. Al-Karaki, R. Ul-Mustafa and A. E. Kamal, "Data Aggregation and Routing in Wireless Sensor Networks: Optimal and Heuristic Algorithms," Computer Networks, Vol. 53, No. 7, 2009, pp. 945-960.

[21] W. M. Lee and V. W. Wong, "E-Span and LPT for Data Aggregation in Wireless Sensor Networks," Computer Communications, Vol. 29, No. 13-14, 2006, pp. 25062520 .

[22] W. Liao, Y. Kao and C. Fan, "Data Aggregation in Wireless Sensor Networks Using Ant Colony Algorithm," Journal of Network and Computer Applications, Vol. 31, No. 4, 2008, pp. 387-401.

[23] S. Ozdemir and Y. Xiao, "Secure Data Aggregation in Wireless Sensor Networks: A Comprehensive Overview," Computer Networks, Vol. 53, No. 12, 2009, pp. 2022-2037.
[24] S. Ozdemir, "Functional Reputation Based Reliable Data Aggregation and Transmission for Wireless Sensor Networks," Computer Communications, Vol. 31, No. 17 2008, pp. 3941-3953.

[25] W. R. Heinzelman, A. Chandrakasan and H. Balakrishnan, "Energy-Efficient Communication Protocol for Wireless Microsensor Networks," Proceedings 33rd Hawaii International Conference System Sciences, Vol. 8, 2000, p. 8020.

[26] A. S. Malik, J. Kuang, J. Liu and W. Chong, "Energy Consumption and Lifetime Analysis in Cluster-Based Wireless Sensor Networks for Periodic Monitoring Applications," Proceedings of the 2009 International Conference on Networks Security, Wireless Communications and Trusted Computing, Wuhan, Vol. 1, 25-26 April 2009, pp. 657-661.

[27] Z. Zhang, "Towards Cluster Based Wireless Sensor Network Deployment Management and Network Coverage Verification," Proceedings of the 11th AsiaPacific Symposium on Network Operations and Management: Challenges For Next Generation Network Operations and Service Management, Beijing, Vol. 5297, 22-24 October 2008, pp. 197-206.

[28] Y. Huang, N. Wang and M. Chen, "Performance of a Hierarchical Cluster-Based Wireless Sensor Network," Proceedings of the 2008 IEEE international Conference on Sensor Networks, Ubiquitous, and Trustworthy Computing, Taichung, 11-13 June 2008, pp. 349-354.

[29] H. Su and X. Zhang, "Optimal Transmission Range for Cluster-Based Wireless Sensor Networks with Mixed Communication Modes," Proceedings of the 2006 international Symposium on World of Wireless, Mobile and Multimedia Networks, Buffalo, 26-29 June 2006, pp. 244-250.

[30] Y. Huang, N. Wang, C. Chen, J. Chen and Z. Guo, "Equalization of Energy Consumption at Cluster Head for Prolonging Lifetime in Cluster-Based Wireless Sensor Networks," WSEAS Transactions on Communications, Vol. 8, No. 5, May 2009, pp. 427-436.

[31] A. T. Hoang and M. Motani, "Collaborative Broadcasting and Compression in Cluster-Based Wireless Sensor Networks," ACM Transactions on Sensor Networks, Vol. 3, No. 3, 2007, p. 17.

[32] Y. Chang, J. Huang and T. Juang, "Dependable Data Aggregation on Cluster-Based Wireless Sensor Networks," Proceedings of the 11th Conference on 11th WSEAS international Conference on Communications, Crete Island, Vol. 11, 26-28 July 2007, pp. 300-305. 


\section{Appendix A}

Algorithm DG

Initialization:

1. $T_{s}(u)=\sum_{k=i}^{n} \sum_{v=j} w l_{(k-n) w+v-w}-l_{0}$

2. $T_{d}(u)=\sum_{k=i+1}^{n} \sum_{v=j+1} w l_{(k-n) w+v-w}-l_{0}$

3. Start $T_{s}(u)$ and $T_{d}(u)$

Transmission Phase:

4. if $T_{s}(u)=0$ then

5. Enter into active state

6. Generate data, and perform data aggregation

7. if $D(u)<p_{i}(F(u)+D(u))$ then

8. Transmit data in direct transmission mode

9. else
10. Transmit data in hop-by-hop transmission mode

11. $T_{s}(u)=\sum_{k=i}^{n} \sum_{v=j} w l_{(k-n) w+v-w}-l_{0}+\tau_{\text {inter } r \text { ounds }}$

12. Start $T_{s}(u)$

13. Enter into sleep state Receiving Phase:

14. if $T_{d}(u)=0$ then

15. Enter into active state

16. $T_{d}(u)=l_{(n-i-1) w+w-j}$, start $T_{d}(u)$

17. Receiving data packets

18. if $T_{d}(u)=0$ then

19. $T_{d}(u)=\sum_{k=i+1}^{n} \sum_{v=j+1} w l_{(k-n) w+v-w}-l_{0}+\tau_{\text {inter } r \text { ounds }}$

20. Enter into sleep state 